\title{
Singularities of logarithmic foliations
}

\author{
Fernando Cukierman, Marcio G. Soares and Israel Vainsencher
}

\section{Abstract}

A logarithmic 1-form on $\mathbb{C P}^{n}$ can be written as

$$
\omega=\left(\prod_{0}^{m} F_{j}\right) \sum_{0}^{m} \lambda_{i} \frac{d F_{i}}{F_{i}}=\lambda_{0} \widehat{F}_{0} d F_{0}+\cdots+\lambda_{m} \widehat{F}_{m} d F_{m}
$$

with $\widehat{F}_{i}=\left(\prod_{0}^{m} F_{j}\right) / F_{i}$ for some homogeneous polynomials $F_{i}$ of degree $d_{i}$ and constants $\lambda_{i} \in \mathbb{C}^{\star}$ such that $\sum \lambda_{i} d_{i}=0$. For general $F_{i}, \lambda_{i}$, the singularities of $\omega$ consist of a schematic union of the codimension 2 subvarieties $F_{i}=F_{j}=0$ together with, possibly, finitely many isolated points. This is the case when all $F_{i}$ are smooth and in general position. In this situation, we give a formula which prescribes the number of isolated singularities.

\section{Introduction and statement of result}

The search for numerical invariants attached to algebraic foliations goes back to Poincaré [Poi91]. He was interested in determining bounds for the degree of curves left invariant by a polynomial vector field on $\mathbb{C}^{2}$.

Recent work has treated the question by establishing relations for the number of singularities of the foliation and certain Chern numbers and then using positivity of certain bundles. For a survey of recent results, see [Bru00, CL91, Est02, Soa00].

A foliation of dimension $r$ on a smooth variety $X$ of dimension $n$ is a coherent subsheaf $\mathcal{F}$ of the tangent sheaf $T X$ of generic rank $r$, locally split in codimension $\geqslant 2$.

If $r=n-1$ (codimension one foliations), the foliation corresponds to a global section of $\Omega_{X}^{1} \otimes \mathcal{L}$ for some line bundle $\mathcal{L}$.

Suppose $X=\mathbb{C P}^{n}$, with homogeneous coordinates $x_{0}, \ldots, x_{n}$. Recall Euler's sequence,

$$
\Omega_{\mathbb{C P}^{n}}^{1}(1) \rightarrow \mathcal{O}^{\oplus n+1} \rightarrow \mathcal{O}(1)
$$

A global section $\omega$ of

$$
\Omega_{\mathbb{C P}^{n}}^{1}(d) \subset \mathcal{O}^{\oplus n+1}(d-1)
$$

can be written as

$$
\omega=\sum_{0}^{n} F_{i} d x_{i}
$$

where $F_{i}$ is a homogeneous polynomial of degree $d-1$, subject to the condition

$$
\sum F_{i} x_{i}=0
$$

(contraction by the radial vector field on $\mathbb{C}^{n+1}$ ).

Received 31 July 2004, accepted in final form 3 January 2005.

2000 Mathematics Subject Classification 32S65, $14 \mathrm{C} 17$.

Keywords: holomorphic foliations, characteristic classes, excess intersection.

The authors were partially supported by CAPES-Brasil, CNPq-Brasil and SPU-Argentina.

This journal is (C) Foundation Compositio Mathematica 2006. 


\section{F. Cukierman, M. G. Soares and I. Vainsencher}

The degree of a codimension one foliation $\mathcal{F}, \operatorname{deg} \mathcal{F}$, is the number of tangencies of the leaves of $\mathcal{F}$ with a generic one-dimensional linear subspace of $\mathbb{C P}^{n}$. A simple calculation shows that $\operatorname{deg} \mathcal{F}=d-2$ if the 1 -form defining $\mathcal{F}$ has components $F_{i}$ of degree $d-1$. The form $\omega$ is integrable if $\omega \wedge d \omega=0$.

Integrable 1-forms make up a Zariski closed subset of $\mathbb{P}\left(H^{0}\left(\Omega^{1}(d)\right)\right.$. We denote by $\operatorname{Fol}\left(\mathbb{C P}^{n} ; d\right)$ the space of codimension one integrable holomorphic foliations of degree $d-2$ of $\mathbb{C P}^{n}$.

Not much is known about the dimensions nor the number of irreducible components of $\operatorname{Fol}\left(\mathbb{C P}^{n} ; d\right)$ (but see [CL96] and [CEL01]).

When $\omega$ can be written as

$$
\omega=\left(\prod_{0}^{m} F_{j}\right) \sum_{0}^{m} \lambda_{i} \frac{d F_{i}}{F_{i}}=\lambda_{0} \widehat{F}_{0} d F_{0}+\cdots+\lambda_{m} \widehat{F}_{m} d F_{m}
$$

for some homogeneous polynomials $F_{i}$ of degree $d_{i}$ and $\lambda_{i} \in \mathbb{C}^{\star}$ such that $\sum \lambda_{i} d_{i}=0$, we say $\omega$ is logarithmic of type $\underline{d}=d_{0}, \ldots, d_{m}$. Given positive integers $d_{0}, \ldots, d_{m}$, set $d=\sum_{i=0}^{m} d_{i}$ and consider the hyperplane

$$
\mathbb{C P}(m-1, \underline{d})=\left\{\left(\lambda_{0}, \ldots, \lambda_{m}\right) \in \mathbb{C P}^{m} \mid \Sigma d_{i} \lambda_{i}=0\right\} .
$$

Define a rational map $\Psi$ by

$$
\begin{aligned}
\mathbb{C P}(m-1, \underline{d}) \times \prod_{i=0}^{m} \mathbb{P}\left(H^{0}\left(\mathbb{C P}^{n}, \mathcal{O}\left(d_{i}\right)\right)\right) \stackrel{\Psi}{\longrightarrow} \operatorname{Fol}\left(\mathbb{C P}^{n} ; d\right) \\
\left(\left(\lambda_{0}, \ldots, \lambda_{m}\right),\left(F_{0}, \ldots, F_{m}\right)\right) \mapsto\left(\prod_{j=0}^{m} F_{j}\right) \sum_{i=0}^{m} \lambda_{i} \frac{d F_{i}}{F_{i}} .
\end{aligned}
$$

The closure of the image of $\Psi$ is the set $\log _{n}(\underline{d})$ of logarithmic foliations of type $\underline{d}$ (of degree $d-2$ ) of $\mathbb{C P}^{n}$. Recall the following result.

Theorem 1 (Calvo-Andrade [Cal94]). For fixed $d_{i}$ and $n \geqslant 3$, logarithmic foliations form an irreducible component of the space of codimension one integrable holomorphic foliations of $\mathbb{C P}^{n}$ of degree $d-2\left(\right.$ with $\left.d=\sum d_{i}\right)$.

The singular scheme of the foliation defined by $\omega \in H^{0}\left(\Omega^{1}(d)\right)$ is the scheme of zeros of $\omega$. This is the closed subscheme with ideal sheaf given by the image of the co-section $\omega^{\vee}:\left(\Omega^{1}(d)\right)^{\vee} \rightarrow \mathcal{O}$.

For $\omega$ general in $H^{0}\left(\Omega^{1}(d)\right)$, there are just finitely many singularities, to wit (cf. Jouanolou [Jou79, Theorem 2.3, p. 87] setting, in his notation, $m=d-1, r=n$ ),

$$
\int_{\mathbb{C P}^{n}} c_{n}\left(\Omega^{1}(d)\right)=\sum_{0}^{n}(-1)^{i}\left(\begin{array}{c}
n+1 \\
i
\end{array}\right) d^{n-i} .
$$

On the other hand of course, a general $\omega$ is not integrable.

Theorem 2 (Jouanolou [Jou79]). For integrable $\omega$, the singular set must contain a codimension 2 component.

It is easy to see that, for logarithmic (hence, integrable) forms

$$
\omega=\lambda_{0} \widehat{F}_{0} d F_{0}+\cdots+\lambda_{m} \widehat{F}_{m} d F_{m}
$$

the singular set contains the union of all codimension two subsets

$$
F_{i}=F_{j}=0, \quad i \neq j .
$$

It is worth mentioning that Jouanolou describes examples of integrable 1-forms with singular schemes containing positive dimensional components of 'wrong' positive dimension. We found no hint as to the existence of isolated singularities for general enough foliations. 


\section{SingularitiES OF LOGARITHMIC FOLIATIONS}

Let $D_{i}$ be the divisor associated to $F_{i}$. We assume that the following genericity conditions hold:

the $D_{i}, i=0, \ldots, m$, are smooth and in general position, $\lambda_{i} \neq 0, i=0, \ldots, m$.

Remark that (1) defines a Zariski open subset of

$$
\mathbb{C P}(m-1, \underline{d}) \times \prod_{i=0}^{m} \mathbb{P}\left(H^{0}\left(\mathbb{C P}^{n}, \mathcal{O}\left(d_{i}\right)\right)\right) .
$$

Before stating our main result recall that the complete symmetric function $\sigma_{\ell}$, of degree $\ell$ in the variables $X_{1}, \ldots, X_{k}$ is defined by: $\sigma_{0}=1$ and, for $\ell \geqslant 1$,

$$
\sigma_{\ell}\left(X_{1}, \ldots, X_{k}\right)=\sum_{i_{1}+\cdots+i_{k}=\ell} X_{1}^{i_{1}} \ldots X_{k}^{i_{k}} .
$$

We then have the following.

Theorem 3. Let $\mathcal{F}$ be a logarithmic foliation on $\mathbb{C P}^{n}$ of type $\underline{d}=d_{0}, \ldots, d_{m}$, given by

$$
\omega=\lambda_{0} \widehat{F}_{0} d F_{0}+\cdots+\lambda_{m} \widehat{F}_{m} d F_{m}
$$

and satisfying (1). Then the singular scheme $S(\mathcal{F})$ of $\mathcal{F}$ can be written as a disjoint union

$$
S(\mathcal{F})=Z \cup R
$$

where

$$
Z=\bigcup_{i<j} D_{i} \cap D_{j}
$$

and $R$ is finite, consisting of

$$
N(n, \underline{d})=\sum_{i=0}^{n}(-1)^{i}\left(\begin{array}{c}
n+1 \\
i
\end{array}\right) \sigma_{n-i}(\underline{d})
$$

points counted with natural multiplicities. Moreover:

(i) $N(n, \underline{d})=0$ if $n \geqslant m$ and $d_{i}=1$ for all $i$;

(ii) $N(n, \underline{d})=\left(\begin{array}{c}m \\ n+1\end{array}\right)$ if $n<m$ and $d_{i}=1$ for all $i$;

(iii) $N(n, \underline{d})>0$ whenever $d_{i} \geqslant 2$ for some $i$.

It will be shown below, see formula (8) in $\S 4.3$, that

$$
N(n, \underline{d})=\text { the coefficient of } h^{n} \text { in } \frac{(1-h)^{n+1}}{\prod_{0}^{m}\left(1-d_{i} h\right)}
$$

from which we deduce the following example.

\subsection{Example}

If $d_{i}=1$ for all $i$ then $(1-h)^{n+1} / \prod_{0}^{m}\left(1-d_{i} h\right)$ reduces to $(1-h)^{n} /(1-h)^{m}$ and we have items (i) and (ii) of the theorem.

(i) $n \geqslant m$. In this case $(1-h)^{n} /(1-h)^{m}$ is a polynomial of degree $n-m<n$ and hence the coefficient of $h^{n}$ vanishes, so that there are no isolated zeros.

(ii) $n<m$. In this case $(1-h)^{n} /(1-h)^{m}$ reads $1 /(1-h)^{m-n}$ and it is easily seen that the coefficient of $h^{n}$ is $\left(\begin{array}{c}m \\ n+1\end{array}\right)$. 


\section{F. Cukierman, M. G. Soares and I. Vainsencher}

\section{Proof of the theorem}

We will show that, if a point is non-isolated in $S(\mathcal{F})$, then it lies in $D_{i} \cap D_{j}$ for some $i<j$. Indeed, let $C$ be an irreducible component of $S(\mathcal{F})$ of dimension $1 \leqslant \operatorname{dim} C \leqslant n-2$. By ampleness and general position, we may pick a point $p \in C$ lying in the intersection of precisely $k$ of the divisors $D_{i}, 1 \leqslant k \leqslant \min \{n, m+1\}$. Let $f_{i}$ be a local equation for $D_{i}$ at $p$. Near $p$, the foliation $\mathcal{F}$ is given by the 1 -form

$$
\varpi=f_{0} \cdots f_{m} \sum_{i=0}^{m} \lambda_{i} \frac{d f_{i}}{f_{i}} .
$$

Renumbering the indices we may assume $p \in D_{0} \cap \cdots \cap D_{k-1}$. The local defining equations $f_{i}=0$ of the $D_{i}$, for $i=0, \ldots, k-1$, are part of a regular system of parameters, i.e. $d f_{0}, \ldots, d f_{k-1}$ are linearly independent at $p$. Write $\widetilde{g}=f_{k} \cdots f_{m}$. Since $p \notin D_{j}, k \leqslant j \leqslant m$, we may assume $\tilde{g}$ vanishes nowhere around $p$ and write $\varpi$ as

$$
\varpi=f_{0} \cdots f_{k-1} \tilde{g}\left[\sum_{j=0}^{k-1} \lambda_{j} \frac{d f_{j}}{f_{j}}+\sum_{i=k}^{m} \lambda_{i} \frac{d f_{i}}{f_{i}}\right]=f_{0} \cdots f_{k-1} \tilde{g}\left[\sum_{j=0}^{k-1} \lambda_{j} \frac{d f_{j}}{f_{j}}+\eta\right],
$$

where $\eta=\sum_{i=k}^{m} \lambda_{i}\left(d f_{i} / f_{i}\right)$ is a holomorphic closed form near $p$. Since $\eta$ is closed, by the formal Poincaré lemma it is exact near $p$, say $\eta=d \xi$. Set $\vartheta=\varpi / \widetilde{g}$. Then $\mathcal{F}$ is defined around $p$ by

$$
\vartheta=f_{0} \cdots f_{k-1}\left[\sum_{j=0}^{k-1} \lambda_{j} \frac{d f_{j}}{f_{j}}+d \xi\right]=f_{0} \cdots f_{k-1}\left[\lambda_{0} \frac{d\left(\exp \left[\xi / \lambda_{0}\right] f_{0}\right)}{\exp \left[\xi / \lambda_{0}\right] f_{0}}+\sum_{j=1}^{k-1} \lambda_{j} \frac{d f_{j}}{f_{j}}\right] .
$$

Set $z_{0}=\exp \left[\xi / \lambda_{0}\right] f_{0}$ and $z_{1}=f_{1}, \ldots, z_{k-1}=f_{k-1}$. Since $u=\exp \left[\xi / \lambda_{0}\right]$ is a unit, we also have that $z_{0}, \ldots, z_{k-1}$ are part of a regular system of parameters at $p$. Now $\vartheta$ can be written as

$$
\vartheta=\frac{z_{0}}{u} z_{1} \cdots z_{k-1}\left[\lambda_{0} \frac{d z_{0}}{z_{0}}+\sum_{j=1}^{k-1} \lambda_{j} \frac{d z_{j}}{z_{j}}\right] .
$$

Thus, $\mathcal{F}$ is defined around $p$ by the 1 -form

$$
\widetilde{\vartheta}=z_{0} z_{1} \cdots z_{k-1}\left[\lambda_{0} \frac{d z_{0}}{z_{0}}+\sum_{j=1}^{k-1} \lambda_{j} \frac{d z_{j}}{z_{j}}\right]=\sum_{j=0}^{k-1} \lambda_{j} z_{0} \cdots \widehat{z_{j}} \cdots z_{k-1} d z_{j} .
$$

If $k=1,(2)$ shows that the foliation is defined near $p$ by $d z_{0}$ and then is non-singular at $p$. Hence, we necessarily have $k \geqslant 2$. Note that the ideal of the scheme of zeros of $\widetilde{\vartheta}$ (as well as of $\omega$ ) near $p$ is generated by the $k$ monomials $z_{0} \cdots \widehat{z_{j}} \cdots z_{k-1}$ with $0 \leqslant j \leqslant k-1$. That is, just the scheme union $\bigcup_{i, j} D_{i} \cap D_{j}$, for $0 \leqslant i<j \leqslant k-1$. Thus, $C$ must be contained in $D_{i} \cap D_{j}$, for some $i<j$, and therefore $C$ is an irreducible component of $D_{i} \cap D_{j}$ and $\operatorname{dim} C=n-2$.

The formula for the finite part is proved in the next section in a slightly more general context.

\subsection{Remark}

The argument above shows that the codimension two part, $Z=\bigcup D_{i j}$, of the singular scheme of a general logarithmic foliation is equal to the singular scheme of the normal crossing divisor $\bigcup D_{i}$. This will enable us to use Aluffi's formula [Alu99] for the Segre class. We also note that, since $D_{i j}$ is smooth and connected, the component $C$ is actually equal to some $D_{i j}$. 


\section{SingularitiEs OF LOGARITHMIC FOLIATIONS}

\section{Formulas}

Let $\mathcal{E} \rightarrow X$ be a holomorphic vector bundle of rank $n$ over a complex projective smooth variety of dimension $n$. Let $s: X \rightarrow \mathcal{E}$ be a section. Assume that:

(1) the scheme of zeros $W$ of $s$ is a disjoint union

$$
W=Z \cup R
$$

with $R$ finite;

(2) there are Cartier divisors $D_{0}, \ldots, D_{m}, m \geqslant 1$, such that

$$
Z=\bigcup_{i<j} D_{i j}
$$

as schemes, where

$$
D_{i j}=D_{i} \cap D_{j}
$$

(3) for all choices of indices

$$
I_{r}=\left(0 \leqslant i_{1}<\cdots<i_{r} \leqslant m\right),
$$

the intersection $D_{I_{r}}=\bigcap_{i \in I_{r}} D_{i}$ is transversal.

We are mainly interested in the case where $X=\mathbb{C P}^{n}$ and the section $s$ is a logarithmic form as in Theorem 3.

We give an expression for the number of points in $R$, counted with natural multiplicities, in terms of the intersection numbers

$$
D^{J} \cdot c_{j}(\mathcal{E})
$$

with

$$
J=\left(j_{0}, \ldots, j_{m}\right), \quad D^{J}=D_{0}^{j_{0}} \cdots D_{m}^{j_{m}}, \quad|J|+j=n .
$$

When $Z=\bigcup_{i<j} D_{i j}$ is a disjoint union, the formula is but a simple direct application of usual excess intersection techniques as reviewed below.

Disjointness implies that $Z$ is a local complete intersection with explicitly known normal bundle.

The ideal of $W$ is the image $\mathcal{I}(W)$ of the co-section

$$
s^{\vee}: \mathcal{E}^{\vee} \rightarrow \mathcal{O} .
$$

It can be written as

$$
\mathcal{I}(W)=\mathcal{I}(Z) \cdot \mathcal{I}(R) .
$$

Locally, it is of the form $\mathcal{I}=\left\langle z_{0}, z_{1}\right\rangle \cdot \mathfrak{m}$, where $z_{0}, z_{1}$ are equations for the pair of transversal divisors cutting $Z$, and $\mathfrak{m}$ denotes an ideal of finite co-length corresponding to the finite part $R \subset W$. (Note that $\mathfrak{m}=\langle 1\rangle$ if $R$ is disjoint from the present coordinate chart.)

Let $\pi: X^{\prime} \rightarrow X$ be the blowup along $Z$. Put $E^{\prime}=\pi^{-1}(Z)$, the exceptional divisor. The pullback $\pi^{\star} s^{\vee}$ of the co-section maps $\pi^{\star} \mathcal{E}^{\vee}$ onto

$$
\mathcal{O}\left(-E^{\prime}\right) \cdot \mathcal{I}\left(R^{\prime}\right)
$$

$\left(R^{\prime}=\pi^{-1} R\right)$. We get an induced map of sheaves

$$
\left(s^{\prime}\right)^{\vee}: \pi^{\star} \mathcal{E}^{\vee} \otimes \mathcal{O}\left(E^{\prime}\right) \rightarrow \mathcal{I}\left(R^{\prime}\right) \subseteq \mathcal{O} .
$$

Dualizing, we find a section $s^{\prime}$ of

$$
\mathcal{E}^{\prime}=\mathcal{E} \otimes \mathcal{O}\left(-E^{\prime}\right)
$$

whose scheme of zeros is precisely $R^{\prime} \simeq R$, the finite part. 


\section{F. Cukierman, M. G. Soares and I. Vainsencher}

Indeed, since $R$ is disjoint from the blowup center, $\pi: X^{\prime} \rightarrow X$ is an isomorphism in a neighborhood of $R^{\prime}$. Hence, the length of $\mathcal{O}_{X^{\prime}} / \mathcal{I}\left(R^{\prime}\right)$ is the same as for $R$. This implies the formula for the degree of the zero cycle,

$$
\operatorname{deg}[R]=\operatorname{deg}\left[R^{\prime}\right]=\int_{X^{\prime}} c_{n}\left(\mathcal{E}^{\prime}\right) .
$$

To compute it explicitly, recall that the exceptional divisor $E^{\prime}$ is the projective bundle $\mathbb{P}\left(\mathcal{N}_{Z / X}\right)$ of the normal bundle of $Z$ in $X$. The restriction of $\mathcal{N}_{Z / X}$ to each $D_{i j}$ is the restriction of $\mathcal{O}\left(D_{i}\right) \oplus \mathcal{O}\left(D_{j}\right)$. Let $\iota: E^{\prime} \hookrightarrow X^{\prime}$ be the inclusion. We recall from [Ful84, B.6, p. 435] a couple of facts that follow from the construction of the blowup as $\operatorname{Proj}\left(\oplus \mathcal{I}^{k}\right)$ of the Rees algebra of the ideal sheaf $\mathcal{I}=\mathcal{I}(Z)$. The natural relatively ample line bundle $\mathcal{O}_{X^{\prime}}(1)$ is presently the image of $\pi^{\star} \mathcal{I} \rightarrow \pi^{\star} \mathcal{O}_{X}=\mathcal{O}_{X^{\prime}}$, thus it is equal to the exceptional ideal sheaf $\mathcal{O}_{X^{\prime}}\left(-E^{\prime}\right)$. The exceptional divisor $E^{\prime} \subset X^{\prime}$ is identified to the projectivization of the normal cone, $\operatorname{Proj}\left(\oplus \mathcal{I}^{k} / \mathcal{I}^{k+1}\right)$. Accordingly, we have the identification $\iota^{\star} \pi^{\star} \mathcal{I}=\mathcal{I} / \mathcal{I}^{2} \rightarrow \iota^{\star} \mathcal{O}_{X^{\prime}}(1)$. The latter is simply the hyperplane bundle $\mathcal{O}_{E^{\prime}}(1)$ of the $\mathbb{C P}^{1}$-bundle $E^{\prime}=\mathbb{P}\left(\mathcal{N}_{Z / X}\right) \rightarrow Z$. We may compute the self-intersection (cf. [Ful84, 2.6, p. 44]),

$$
\begin{aligned}
\left(E^{\prime}\right)^{2} & =\iota_{\star}\left(\iota^{\star} E^{\prime}\right)=\iota_{\star}\left(\iota^{\star} c_{1}\left(\mathcal{O}_{X^{\prime}}\left(E^{\prime}\right)\right) \cap\left[X^{\prime}\right]\right) \\
& =\iota_{\star}\left(\iota^{\star} c_{1}\left(\mathcal{O}_{X^{\prime}}(-1)\right) \cap\left[X^{\prime}\right]\right) \\
& =-\iota_{\star}\left(\xi \cap\left[E^{\prime}\right]\right)
\end{aligned}
$$

with

$$
\xi=c_{1}\left(\mathcal{O}_{E^{\prime}}(1)\right)
$$

Recall that the push-forward of powers of the hyperplane class $\xi$ of the $\mathbb{C P}^{1}$-bundle $E^{\prime}=$ $\mathbb{P}\left(\mathcal{N}_{Z / X}\right) \rightarrow Z$ are expressed (cf. [Ful84, p. 47]) by Segre classes:

$$
\pi_{\star}\left(\xi^{j+1}\right)=s_{j}\left(\mathcal{N}_{Z / X}\right) \quad \forall j \in \mathbb{Z} .
$$

Writing $\left[D_{i j}\right]$ for the cycle class of $D_{i} \cap D_{j}$ in the Chow (or homology) group $A_{\star} X$, we have, for $r \geqslant 0$,

We may write

$$
\left(E^{\prime}\right)^{r+1}=\iota_{\star}\left(\iota^{\star}\left(E^{\prime}\right)^{r}\right)=\iota_{\star}\left((-\xi)^{r} \cap\left[E^{\prime}\right]\right) .
$$

$$
\begin{aligned}
\pi_{\star}\left(\left(E^{\prime}\right)^{r+1}\right) & =\pi_{\star} \iota_{\star}\left((-\xi)^{r} \cap\left[E^{\prime}\right]\right) \\
& =(-1)^{r} \sum_{i<j} s_{r-1}\left(\mathcal{O}\left(D_{i}\right) \oplus \mathcal{O}\left(D_{j}\right)\right) \cap\left[D_{i j}\right]
\end{aligned}
$$

in the group $A_{m} X$ of cycles of dimension $m=n-2-k$.

Put

$$
\begin{aligned}
s_{k i j} & =s_{k}\left(\mathcal{O}\left(D_{i}\right) \oplus \mathcal{O}\left(D_{j}\right)\right) \cap\left[D_{i j}\right] \\
& =(-1)^{k} D_{i} \cdot D_{j} \cdot \sum_{u=0}^{k} D_{i}^{u} D_{j}^{k-u} .
\end{aligned}
$$

Since $s_{j}=0$ for $j<0$, we also have

$$
\pi_{\star}\left(\left(E^{\prime}\right)\right)=0 .
$$

It follows from (4) and (3) that

$$
\begin{aligned}
\operatorname{deg}[R] & =\int_{X} \pi_{\star} c_{n}\left(\mathcal{E}^{\prime}\right) \\
& =\int_{X} \sum_{r=0}^{n} c_{n-r}(\mathcal{E}) \cdot \pi_{\star}\left(\left(-E^{\prime}\right)^{r}\right)
\end{aligned}
$$




$$
\begin{aligned}
& =\int_{X} c_{n}(\mathcal{E})+\sum_{r=1}^{n-1}(-1)^{r+1} c_{n-1-r}(\mathcal{E}) \cdot \pi_{\star}\left(\left(E^{\prime}\right)^{r+1}\right) \\
& =\int_{X} c_{n}(\mathcal{E})-\sum_{r=1}^{n-1} \sum_{i<j} c_{n-1-r}(\mathcal{E}) \cdot s_{(r-1) i j} \\
& =\int_{X} c_{n}(\mathcal{E})-\sum_{r=1}^{n-1}(-1)^{r-1} c_{n-1-r}(\mathcal{E}) \sum_{i<j} \sum_{u=0}^{r-1} D_{i}^{u+1} D_{j}^{r-u} .
\end{aligned}
$$

The idea now is to reduce the general case to the above situation. This will be done by a sequence of blowups along smooth centers with known normal bundles.

We explain how the reduction works, say in the case when all four-fold intersections are empty, for the sake of simplicity. The general case is entirely similar. Thus assume that for all

$$
I_{4}=\left(0 \leqslant i_{0}<i_{2}<i_{3}<i_{4} \leqslant m\right),
$$

we have

$$
D_{I_{4}}:=\bigcap_{i \in I_{4}} D_{i}=\emptyset
$$

(This is the case if, for instance, $\operatorname{dim} X=3$.) It follows that for all choices of triple indices,

$$
I_{3}=(i<j<k) \neq I_{3}^{\prime}=\left(i^{\prime}<j^{\prime}<k^{\prime}\right) \text {, }
$$

we must have

$$
D_{I_{3}} \cap D_{I_{3}^{\prime}}=\emptyset \text {. }
$$

Now, the union $T$ of all triple intersections $D_{I_{3}}$ is smooth.

Let $\pi: X^{\prime} \rightarrow X$ be the blowup along $T$. The strict transform $D_{i j}^{\prime}$ is equal to the blowup of $D_{i j}$ along the disjoint union of Cartier divisors $D_{i j k}$, hence $D_{i j}^{\prime} \simeq D_{i j}$ holds. Moreover, since $D_{i j} \cap D_{j k}$ is a union of connected components of the blowup center, it follows that $D_{i j}^{\prime} \cap D_{j k}^{\prime}=\emptyset$. We also have that the $D_{i}^{\prime}$ meet transversally.

Look at the pullback $\pi^{-1} W$ of the zero scheme of the section $s$. We will take coordinates on $X$ in a neighborhood of a point $0 \in D_{123}$, say. Near $0, W$ is equal to the union $D_{12} \cup D_{13} \cup D_{23}$. Let $z_{i}=0$ be a local equation of $D_{i}$. Then the ideal of $W$ near 0 is equal to the intersection

$$
\left\langle z_{1}, z_{2}\right\rangle \cap\left\langle z_{1}, z_{3}\right\rangle \cap\left\langle z_{2}, z_{3}\right\rangle=\left\langle z_{1} z_{2}, z_{1} z_{3}, z_{2} z_{3}\right\rangle
$$

The blowup center, $T$, is locally given by $\left\langle z_{1}, z_{2}, z_{3}\right\rangle$. The restriction of $X^{\prime}$ over the present affine neighborhood of the point 0 is covered by three affine open subsets, one for each choice of $z_{i}$ as a generator of the exceptional ideal $\mathcal{O}\left(-E^{\prime}\right)$.

Say we take $z_{1}$ as a local generator. We may write $z_{i}=z_{1} z_{i}^{\prime}, i=1,2$. Here $z_{i}^{\prime}$ is a local equation of the strict transform of $D_{i}$.

The pullback of $W$ is given by the ideal

$$
\mathcal{I}\left(\pi^{-1} W\right)=z_{1}^{2}\left\langle z_{2}^{\prime}, z_{3}^{\prime}, z_{2}^{\prime} z_{3}^{\prime}\right\rangle=z_{1}^{2}\left\langle z_{2}^{\prime}, z_{3}^{\prime}\right\rangle .
$$

This is twice the exceptional ideal, times the ideal of the strict transform of $D_{23}$.

Note that the strict transforms of $D_{13}$ and of $D_{12}$ are empty in the present neighborhood of $X^{\prime}$. Thus, the $D_{i j}^{\prime}$ are presently disjoint.

The local expression shows that the image $\mathcal{I}(W) \mathcal{O}_{X^{\prime}}$ of the co-section

$$
\pi^{\star} s^{\vee}: \mathcal{E}^{\vee} \rightarrow \mathcal{O}_{X^{\prime}}
$$




\section{F. Cukierman, M. G. Soares and I. Vainsencher}

is of the form

$$
\mathcal{I}(W) \mathcal{O}_{X^{\prime}}=\mathcal{O}\left(-2 E^{\prime}\right) \cdot \mathcal{I}\left(Z^{\prime}\right) \cdot \mathcal{I}\left(R^{\prime}\right),
$$

where the finite piece $R^{\prime}=\pi^{-1}(R) \simeq R$ and $Z^{\prime}=\bigcup D_{i j}^{\prime}$ is the disjoint union of pairwise transversal intersections of Cartier divisors $D_{i}^{\prime}$.

Hence, we may apply the previous case to the section $s^{\prime}=s \otimes \mathcal{O}\left(-2 E^{\prime}\right)$ of $\mathcal{E}^{\prime}=\mathcal{E} \otimes \mathcal{O}\left(-2 E^{\prime}\right)$. We find

$$
\begin{aligned}
\operatorname{deg}[R] & =\operatorname{deg}\left[R^{\prime}\right] \\
& =\int_{X^{\prime}} c_{n}\left(\mathcal{E}^{\prime}\right)-\sum_{r=0}^{n-1}(-1)^{r-1} c_{n-1-r}\left(\mathcal{E}^{\prime}\right) \sum_{i<j} \sum_{u=0}^{r-1}\left(D_{i}^{\prime}\right)^{u+1} \cdot\left(D_{j}^{\prime}\right)^{r-u} .
\end{aligned}
$$

Let $E_{i}^{\prime}$ denote the sum of the (disjoint) exceptional divisors over all $D_{I_{3}}$ with $i \in I_{3}$. Using the formulas $D_{i}^{\prime}=\pi^{\star} D_{i}-E_{i}^{\prime}$ and universal formulas for $c\left(\mathcal{E} \otimes \mathcal{O}\left(-2 E^{\prime}\right)\right)$ and applying $\pi_{\star}$, the above expression can be written in terms of the intersection numbers $D^{J} \cdot c_{j}(\mathcal{E})$.

In general, let $r$ be the smallest integer such that for all possible choices of indices

$$
I_{r+2}=\left(0 \leqslant i_{0}<i_{1}<\cdots<i_{r+1} \leqslant m\right),
$$

we have

$$
D_{I_{r+2}}:=\bigcap_{i \in I_{r+2}} D_{i}=\emptyset .
$$

If $m \geqslant 2$, we have $r \leqslant \min (n-1, m-1)$ because $\operatorname{dim} X=n$ and the divisors are in general position. Of course, if $r \geqslant m$ no $I_{r+2}$ exists! If $m=1$, set $r=1$.

We then have that the union

$$
Z_{r+1}=\bigcup_{I_{r+1}} D_{I_{r+1}}
$$

of all $(r+1)$-fold intersections among $D_{i}$ is smooth. Let $\pi^{1}: X^{1} \rightarrow X$ be the blowup along $Z_{r+1}$. A local analysis as performed above shows that the strict transforms $D_{i}^{1}$ are in general position and the intersections $D_{I_{r+1}}^{1}$ are empty. Moreover, there is a section $s^{1}$ of $\mathcal{E}^{1}=\mathcal{E} \otimes \mathcal{O}\left(-r E^{1}\right)$ with zeros scheme $W^{1}$ equal to the disjoint union $Z^{1} \cup R^{1}$, with $R^{1}=\left(\pi^{1}\right)^{-1}(R) \simeq R$. Here $Z^{1}$ is the scheme union of the pairwise intersections $D_{i j}^{1}$. Continuing this way, we construct a sequence of blowups,

$$
X^{r} \stackrel{\pi^{r}}{\longrightarrow} \cdots \stackrel{\pi^{2}}{\longrightarrow} X^{1} \stackrel{\pi^{1}}{\longrightarrow} X
$$

such that ultimately the bundle

$$
\mathcal{E}^{r}=\mathcal{E} \otimes \mathcal{O}\left(-r E^{1}-(r-1) E^{2}-\cdots-E^{r}\right)
$$

is endowed with a section $s^{r}$ whose scheme of zeros is exactly

$$
R^{r}=\left(\pi^{r}\right)^{-1} \cdots\left(\pi^{2}\right)^{-1}\left(\pi^{1}\right)^{-1}(R) \simeq R
$$

Thus, we get the formula

$$
\operatorname{deg}(R)=\int_{X} \pi_{\star}^{1} \cdots \pi_{\star}^{r}\left(c_{n}\left(\mathcal{E}^{r}\right)\right)
$$

The right-hand side may clearly be written in terms of the intersection numbers $D^{J} \cdot c_{j}(\mathcal{E})$. 


\section{SiNGULARITIES OF LOGARITHMIC FOLIATIONS}

\section{Examples}

Set for short $c_{i}=c_{i} \mathcal{E}$. Let

$$
\sigma_{i}=\sigma_{i}(\underline{D})=\sum_{i_{0}+\cdots+i_{m}=i} D_{0}^{i_{0}} \cdots D_{m}^{i_{m}}
$$

denote the sum of all monomials of degree $i$ in the classes of the $D_{i}$.

\section{$4.1 m=1$}

We find

$n=3: \quad \operatorname{deg}(R)=c_{3}-D_{0} D_{1} c_{1}+D_{0}^{2} D_{1}+D_{0} D_{1}^{2}$.

$n=4: \quad \operatorname{deg}(R)=c_{4}-D_{0} D_{1} c_{2}+\left(D_{0}^{2} D_{1}+D_{0} D_{1}^{2}\right) c_{1}-\left(D_{0}^{3} D_{1}+D_{0}^{2} D_{1}^{2}+D_{0} D_{1}^{3}\right)$.

These first few cases suggest the formula for general $n$, still with $m=1$,

$$
\operatorname{deg}(R)=c_{n}-\sum_{1}^{n-2}(-1)^{n-i} \sigma_{n-i}(\underline{D}) c_{i}-(-1)^{n} \sigma_{n}(\underline{D}),
$$

which will be generalized in the sequel.

\subsection{Aluffi's formula}

This was explained to us by P. Aluffi. In fact, nearly closed formula can be achieved using Fulton's residual intersection formula (RIF) [Ful84, 9.2.3, p. 163], instead of the above blowup sequence. It requires the knowledge of the Segre class of the excess locus $Z=\bigcup D_{i j}$. This is rendered feasible thanks to Aluffi's formula for the Segre class of the singular scheme of a normal crossing divisor $D=\sum D_{i}$ (cf. [Alu99, proof of Lemma II.2]). The formula reads

$$
s(Z, X)=\left(\left(1-\frac{1-D}{\prod_{0}^{m}\left(1-D_{i}\right)}\right) \cap[X]\right) \otimes_{X} \mathcal{O}(D) .
$$

The right-hand side uses Aluffi's $\bullet \otimes L$ operation on the Chow group introduced in [Alu94]: if $a_{i}$ is a class of codimension $i$ in the Chow group, and $L$ is a line bundle, then

$$
a_{i} \otimes L=\frac{a_{i}}{c(L)^{i}} .
$$

We have

$$
s(Z, X)=[X]-\left(\left(\frac{1-D}{\prod_{0}^{m}\left(1-D_{i}\right)}\right) \cap[X]\right) \otimes_{X} \mathcal{O}(D) .
$$

The operation $\cdot \otimes L$ behaves well with respect to Chern classes of 'rank 0 bundles'(!). That is, if $E, F$ are bundles of the same rank, then

$$
((c(E) / c(F)) \cap a) \otimes L=(c(E \otimes L) / c(F \otimes L)) \cap(a \otimes L) .
$$

We have to pretend that the fraction in (6) is the quotient of the Chern classes of two bundles of the same rank, so regard the second piece as

$$
\left(\frac{(1-D) \cdot 1^{m}}{\prod_{0}^{m}\left(1-D_{i}\right)} \cap[X]\right) \otimes_{X} O(D)
$$

that is, view the numerator as the Chern class of the bundle $\mathcal{O}(-D) \oplus \mathcal{O}^{\oplus m}$. Tensoring by $\mathcal{O}(D)$, the numerator turns from

$$
(1-D) \cdot 1^{m}, \quad \text { into }(1-D+D)(1+D)^{m}=(1+D)^{m} ;
$$




\section{F. Cukierman, M. G. Soares and I. Vainsencher}

the denominator goes from $\prod\left(1-D_{i}\right)$ to $\prod\left(1+D-D_{i}\right)$; and again nothing happens to the term $[X]$, because it is of codimension 0 . Bottom line,

$$
s(Z, X)=[X]-\frac{(1+D)^{m}}{\prod_{0}^{m}\left(1+D-D_{i}\right)} \cap[X] .
$$

We apply Fulton's RIF, in his notation, to the regular embedding $i: X \rightarrow Y$ with $X$ as above, and $i$ equal to the zero section of $Y:=\mathcal{E}$; we take for $f: V=X \rightarrow Y=\mathcal{E}$ the given section $s$ as in the beginning of $\S 3$. Now we have, in one hand, $X \cdot V=c_{n}(\mathcal{E})$ by [Ful84, Example 3.3.2, p. 67 or 6.3 .4$, p. 105]. Presently, the residual intersection class $\mathbb{R}$ is equal to the class of the finite part $R$ since the latter is disjoint from $Z$. Hence, we may write

$$
[\mathbb{R}]=c_{n}(\mathcal{E}) \cap[X]-[c(\mathcal{E}) \cap s(Z, X)]_{n},
$$

where $[\cdot]_{n}$ denotes the $n$-codimensional part of a cycle. We get,

$$
\begin{aligned}
{[\mathbb{R}] } & =[c(\mathcal{E}) \cap([X]-s(Z, X))]_{n} \\
& =c(\mathcal{E}) \cap\left[\frac{(1+D)^{m}}{\prod_{0}^{m}\left(1+D-D_{i}\right)}\right]_{n} .
\end{aligned}
$$

Hence,

$$
\operatorname{deg} R=\int_{X}\left[\frac{c(\mathcal{E})(1+D)^{m}}{\prod_{0}^{m}\left(1+D-D_{i}\right)}\right]
$$

4.2.1 Remark. Let us recall a nice observation in [AF95] to the effect that, if $F$ is a virtual sheaf of rank $n-1$ then $c_{n}(F \otimes L)=c_{n}(F)$ for any line bundle $L$. We may write

$$
\begin{aligned}
\frac{c(\mathcal{E})(1+D)^{m}}{\prod_{0}^{m}\left(1+D-D_{i}\right)} & =c\left(\mathcal{E}+\mathcal{O}(D)^{\oplus m}-\bigoplus_{0}^{m} \mathcal{O}\left(D-D_{i}\right)\right) \\
& =c\left((\underbrace{\mathcal{E} \otimes \mathcal{O}(-D)+\mathcal{O}^{\oplus m}-\bigoplus_{0}^{m} \mathcal{O}\left(-D_{i}\right)}_{\text {rank }=n-1}) \otimes \mathcal{O}(D)\right) .
\end{aligned}
$$

Thus, in degree $n$ we find

$$
\left[\frac{c(\mathcal{E})(1+D)^{m}}{\prod_{0}^{m}\left(1+D-D_{i}\right)}\right]_{n}=\left[\frac{c(\mathcal{E} \otimes \mathcal{O}(-D))}{\prod_{0}^{m}\left(1-D_{i}\right)}\right]_{n}
$$

This can be expanded as

$$
\sum_{0}^{n} c_{i}(\mathcal{E} \otimes \mathcal{O}(-D)) \sigma_{n-i}(\underline{D})=\sum_{0}^{n} \sum_{0}^{i}\left(\begin{array}{c}
n-j \\
i-j
\end{array}\right) c_{j}(\mathcal{E})(-D)^{i-j} \sigma_{n-i}(\underline{D}) .
$$

4.2.2 Remark. The preprint by Catanese et al. [CHKS04] also contains a similar formula, deduced by different methods and in the context of another subject, namely, algebraic statistics.

\subsection{Foliations on $\mathbb{C P}^{n}$}

For $\mathcal{E}=\Omega_{\mathbb{C P}^{n}}^{1}(d)$, the above reduces to

$$
\operatorname{deg} R=\text { coefficient of } h^{n} \text { in } \frac{(1-h)^{n+1}}{\prod_{0}^{m}\left(1-d_{i} h\right)}=\sum_{i=0}^{n}(-1)^{i}\left(\begin{array}{c}
n+1 \\
i
\end{array}\right) \sigma_{n-i}(\underline{d}) .
$$

with $\sigma_{n-i}$ the complete symmetric function of degree $n-i$ in $d_{0}, \ldots, d_{m}$.

One further application of Remark 4.2.1 yields the following positivity result. 


\section{SingulARITIES OF LOGARITHMIC FOLIATIONS}

Proposition 4.4. Assume at least one $d_{i} \geqslant 2$ (and, of course, all $d_{i} \geqslant 1$ ). Then we have $\operatorname{deg} R>0$.

Proof. We show that, under the change of variables $d_{i}=e_{i}+1$, the formula (8) becomes

$$
\operatorname{deg} R=\sum_{0}^{n}\left(\begin{array}{c}
m-1 \\
i
\end{array}\right) \sigma_{n-i}(\underline{e}) .
$$

The latter is obviously $>0$ if some $e_{i}>0$. To show the last equality, we use Remark 4.2.1 to write

$$
\begin{aligned}
c_{n}(\mathcal{O} & \left.(-h)^{\oplus n+1}-\bigoplus_{0}^{m} \mathcal{O}\left(-d_{i} h\right)+\mathcal{O}^{\oplus m-1}\right) \\
& =c_{n}\left(\mathcal{O}^{\oplus n+1}-\bigoplus_{0}^{m} \mathcal{O}\left(h-d_{i} h\right)+\mathcal{O}(h)^{\oplus m-1}\right) \\
& =\left[c\left(\mathcal{O}(h)^{\oplus m-1}-\bigoplus_{0}^{m} \mathcal{O}\left(h-d_{i} h\right)\right)\right]_{n} \\
& =\left[\frac{(1+h)^{m-1}}{\prod_{0}^{m}\left(1-e_{i} h\right)}\right]_{n} \\
& =\sigma_{n}(\underline{e})+(m-1) \sigma_{n-1}(\underline{e})+\left(\begin{array}{c}
m-1 \\
2
\end{array}\right) \sigma_{n-2}(\underline{e})+\cdots
\end{aligned}
$$

\section{ACKNowledgements}

We are grateful to J. V. Pereira and to P. Aluffi for very helpful conversations. We also thank the referee for his suggestions.

\section{REFERENCES}

Alu94 P. Aluffi, MacPherson's and Fulton's Chern classes of hypersurfaces, Internat. Math. Res. Notices 11 (1994), 455-465.

Alu99 P. Aluffi, Chern classes for singular hypersurfaces, Trans. Amer. Math. Soc. 351 (1999), 3989-4026.

AF95 P. Aluffi and C. Faber, A remark on the Chern class of a tensor product, Manuscripta Math. 88 (1995), 85-86.

Bru00 M. Brunella, Birational geometry of foliations, Monografias de Matemática (IMPA, Rio de Janeiro, 2000).

Cal94 O. Calvo-Andrade, Irreducible components of the space of foliations, Math. Ann. 299 (1994), $751-767$.

CHKS04 F. Catanese, S. Hoşten, A. Khetan and B. Sturmfels, The maximum likelihood degree, Preprint (2004), arXiv:math.AG/0406533.

CL91 D. Cerveau and A. Lins Neto, Holomorphic foliations in $\mathbb{C P}^{2}$ having an invariant algebraic curve, Ann. Inst. Fourier 41 (1991), 883-903.

CL96 D. Cerveau and A. Lins Neto, Irreducible components of the space of holomorphic foliations of degree two in $\mathbb{C P}^{n}, n>3$, Ann. of Math. (2) 143 (1996), 577-612.

CEL01 D. Cerveau, S. J. Edixhoven and A. Lins Neto, Pull-back components of the space of holomorphic foliations on $\mathbb{C P}^{n}, n>3$, J. Algebraic Geom. 10 (2001), 695-711.

Est02 E. Esteves, The Castelnuovo-Mumford regularity of an integral variety of a vector field on projective space, Math. Res. Lett. 9 (2002), 1-15.

Ful84 W. Fulton, Intersection theory, Ergeb. Math. Grenzgeb. (3), vol. 2 (Springer, Berlin, 1984). 
Jou79 J. P. Jouanolou, Équations de Pfaff algébriques, Lecture Notes in Mathematics, vol. 708 (Springer, Berlin, 1979).

Poi91 H. Poincaré, Sur l'intégration algébrique des équations différentielles du premier ordre et du premier degré, Rend. Circ. Mat. Palermo (2) 5 (1891), 161-191.

Soa00 M. G. Soares, Projective varieties invariant by one-dimensional foliations, Ann. of Math. (2) 152 (2000), 369-382.

Fernando Cukierman fcukier@dm.uba.ar

Departamento Matematica, FCEyN UBA, Ciudad Universitaria, 1428 Ciudad de Buenos Aires, Argentina

Marcio G. Soares msoares@mat.ufmg.br

Departamento Matemática, UFMG, Avenida Antônio Carlos 6627, 31270-901 Belo Horizonte, Brazil

Israel Vainsencher israel@mat.ufmg.br

Departamento Matemática, UFMG, Avenida Antônio Carlos 6627, 31270-901 Belo Horizonte, Brazil 